\title{
Gj 3236: A New Bright, Very Low Mass Eclipsing Binary System Discovered by the Mearth Observatory
}

\section{Citation}

Irwin, Jonathan, David Charbonneau, Zachory K. Berta, Samuel N. Quinn, David W. Latham, Guillermo Torres, Cullen H. Blake, et al. 2009. "GJ 3236: A NEW BRIGHT, VERY LOW MASS ECLIPSING BINARY SYSTEM DISCOVERED BY THE MEARTH OBSERVATORY." The Astrophysical Journal 701 (2): 1436-49. https://doi.org/10.1088/0004-637x/701/2/1436.

\section{Permanent link}

http://nrs.harvard.edu/urn-3:HUL.InstRepos:41397458

\section{Terms of Use}

This article was downloaded from Harvard University's DASH repository, and is made available under the terms and conditions applicable to Other Posted Material, as set forth at http:// nrs.harvard.edu/urn-3:HUL.InstRepos:dash.current.terms-of-use\#LAA

\section{Share Your Story}

The Harvard community has made this article openly available.

Please share how this access benefits you. Submit a story.

\section{Accessibility}




\title{
GJ 3236: A NEW BRIGHT, VERY LOW MASS ECLIPSING BINARY SYSTEM DISCOVERED BY THE MEARTH OBSERVATORY
}

\author{
Jonathan Irwin ${ }^{1}$, David Charbonneau ${ }^{1}$, Zachory K. Berta $^{1}$, Samuel N. Quinn $^{1}$, David W. Latham ${ }^{1}$, \\ Guillermo Torres ${ }^{1}$, Cullen H. Blake ${ }^{1}$, Christopher J. Burke ${ }^{1}$, Gilbert A. Esquerdo ${ }^{1}$, Gábor Fürész ${ }^{1}$, \\ Douglas J. Mink ${ }^{1}$, Philip Nutzman ${ }^{1}$, Andrew H. Szentgyorgyi ${ }^{1}$, Michael L. Calkins ${ }^{2}$, Emilio E. Falco ${ }^{2}$, \\ Joshua S. BLOOM ${ }^{3,4}$, AND DAN L. STARR ${ }^{3}$ \\ ${ }^{1}$ Harvard-Smithsonian Center for Astrophysics, 60 Garden Street, Cambridge, MA 02138, USA; jirwin@ @fa.harvard.edu \\ ${ }^{2}$ Fred Lawrence Whipple Observatory, Smithsonian Astrophysical Observatory, 670 Mount Hopkins Road, Amado, AZ 85645, USA \\ ${ }^{3}$ Astronomy Department, University of California, 445 Campbell Hall, Berkeley, CA 94720, USA \\ Received 2009 May 26; accepted 2009 June 25; published 2009 July 31
}

\begin{abstract}
We report the detection of eclipses in GJ 3236, a bright $(I=11.6)$, very low mass binary system with an orbital period of 0.77 days. Analysis of light and radial velocity curves of the system yielded component masses of $0.38 \pm 0.02 M_{\odot}$ and $0.28 \pm 0.02 M_{\odot}$. The central values for the stellar radii are larger than the theoretical models predict for these masses, in agreement with the results for existing eclipsing binaries, although the present 5\% observational uncertainties limit the significance of the larger radii to approximately $1 \sigma$. Degeneracies in the light curve models resulting from the unknown configuration of surface spots on the components of GJ 3236 currently dominate the uncertainties in the radii, and could be reduced by obtaining precise, multiband photometry covering the full orbital period. The system appears to be tidally synchronized and shows signs of high activity levels as expected for such a short orbital period, evidenced by strong $\mathrm{H} \alpha$ emission lines in the spectra of both components. These observations probe an important region of mass-radius parameter space around the predicted transition to fully convective stellar interiors, where there are a limited number of precise measurements available in the literature.
\end{abstract}

Key words: binaries: eclipsing - stars: individual (GJ 3236) - stars: low-mass, brown dwarfs

Online-only material: color figures, machine-readable tables

\section{INTRODUCTION}

Detached, double-lined eclipsing binaries provide a largely model-independent means to precisely and accurately measure fundamental stellar properties, particularly masses and radii. In the best-observed systems, the precision of these can be at the $<1 \%$ level, and thus place stringent constraints on stellar evolution models (e.g., Andersen 1991).

Despite this, as far as we are aware, there are at present only four known systems with one or more components between $0.4 M_{\odot}$ and the hydrogen-burning limit on the main sequence (i.e., old, field stars): CM Dra (Eggen \& Sandage 1967; Lacy 1977; Metcalfe et al. 1996; Morales et al. 2009), CU Cnc B (Delfosse et al. 1999; Ribas 2003), LP 133-373 (Vaccaro et al. 2007), and SDSS J031824-010018 (Blake et al. 2008). Although JW 380 (Irwin et al. 2007b), NSVS 02502726B (Cakirli et al. 2009), and the NGC 1647 system of Hebb et al. (2007) also satisfy this mass criterion, these objects are still contracting on the pre-main sequence.

Of the known systems, the only two with parameters determined to better than 2\% are CM Dra and CU Cnc. For SDSS J031824-010018, the knowledge of the parameters is limited largely by radial velocity errors, since this system is extremely faint (SDSS $r=19.3$ ) and has an extremely short orbital period (0.41 days), meaning long integrations cannot be used to obtain better signal to noise. Hence, there is little possibility for substantial improvement in the parameters in the near future. It is clear that, in order to better constrain the stellar mass-radius relation on the main sequence, more bright, low-mass eclipsing binaries are needed to yield extremely precise masses and radii.

\footnotetext{
4 Sloan Research Fellow
}

Furthermore, observations of these systems have indicated significant discrepancies with the stellar models. This is particularly the case below $0.4 M_{\odot}$, and the components of CM Dra (the lowest-mass system with $<1 \%$ observational errors) have radii $10 \%-15 \%$ larger than the theoretical predictions from stateof-the-art stellar evolution models. It has been suggested (e.g., Chabrier et al. 2007) that the reason for this discrepancy may be that close binaries are not actually representative of single stars at this level of precision. The effect of the close companion and tidal locking is likely to significantly increase activity levels in close binaries, and these authors suggest that it could be this effect that is responsible for the inflated radii of CM Dra.

López-Morales (2007) examined the available sample of single-star and eclipsing binary measurements to search for correlations of radius with activity levels and metallicity, finding that such a correlation of activity with radius does appear to exist for members of close binary stars. This result was based on a small number of measurements for late $\mathrm{M}$ dwarfs, and the conclusions would be strengthened by the availability of additional precise estimates of the parameters for binaries with a range of activity levels and orbital periods, to explore the available parameter space.

We present the discovery of a new bright, low-mass eclipsing binary system, GJ 3236. The components show high activity levels, as evidenced by $\mathrm{H} \alpha$ emission lines in the spectra of both stars, and show rotational modulations in the light curves that are synchronized with the binary orbital period of 0.77 days. This system thus has the potential to yield an additional precise test of stellar evolution models for high activity and short orbital periods. 


\section{OBSERVATIONS AND DATA REDUCTION}

\subsection{MEarth Photometry}

Eclipses in GJ 3236 were detected during 2008 January in the first two weeks of routine operations of the newly commissioned MEarth observatory, a system designed primarily to search for transiting super-Earth exoplanets orbiting around the nearest 2000 mid- to late-M dwarfs in the northern hemisphere (Nutzman \& Charbonneau 2008; Irwin et al. 2009). Exposure times on each field observed by MEarth are tailored to achieve sensitivity to a particular planet size for the assumed stellar parameters of the target star and were $82 \mathrm{~s}$ for GJ 3236.

After the initial detection of the eclipses, we switched to a follow-up mode, observing at the highest possible cadence (i.e., continuously, resulting in a cadence of approximately 2 minutes including overheads) during eclipse and for an additional $1 \mathrm{hr}$ window surrounding the eclipse, and at the normal cadence for the MEarth survey of approximately 20 minutes for the remainder of the time. In this way we sample, as well as possible, the rapid flux decrement during eclipse, and the out-of-eclipse portions of the light curve. We note that sampling the latter is of crucial importance for obtaining accurate parameters from light curve analyses of binary systems showing out-of-eclipse modulations, such as the present one.

We obtained data both in the initial season after the eclipses were first detected (2008 January to 2008 May, inclusive), and following the summer monsoon (2008 October onward). During this time, there were a number of software improvements (including the ability to update the telescope pointing after taking each science image, resulting in substantially improved light curves due to minimizing the drift across the detector, and hence the effect of flat-fielding errors, fringing, etc.) and our filter system was changed from Cousins $I$ to a custom longpass filter with transmission from $715 \mathrm{~nm}$, and limited at the long wavelength end by the tail of the CCD quantum efficiency curve (see Nutzman \& Charbonneau 2008). We have elected to use only the latter data set for modeling, despite this having the disadvantage of being observed in a non-standard bandpass, because it is approximately contemporaneous with our $V$-band follow-up photometry. We see evidence for evolution of the amplitude and phase of the out-of-eclipse modulation between the two observing seasons, so this simplifies the analysis by allowing the use of a single set of spot parameters to describe both bandpasses.

A total of 1540 observations were obtained between UT 2008 October 2 and UT 2008 December 11, including six primary and three secondary eclipses sampled at high cadence.

Data were reduced using the standard MEarth reduction pipeline, which is at present identical to the Monitor project pipeline described in Irwin et al. (2007a). We used a smaller aperture radius of 5 pixels (3". 8 ) than the usual value of 10 pixels adopted for bright stars, due to the presence of a nearby star approximately $7^{\prime \prime}$ from GJ 3236 at the epoch of our observations, and 4 mag fainter in the MEarth bandpass. This star does not share a common proper motion with GJ 3236 and is therefore not physically associated. Reducing the aperture size in this way reduces the flux contributed by this star in the light curve of GJ 3236 to a negligible level (we estimate a $0.1 \%$ error in the measured fluxes, and thus also in the eclipse depths).

The high proper motion of the GJ 3236 system allows us to constrain the contribution of any additional background stars in the photometric aperture that are not comoving with GJ 3236 itself, using previous epochs of imaging. We show in Figure 1 a

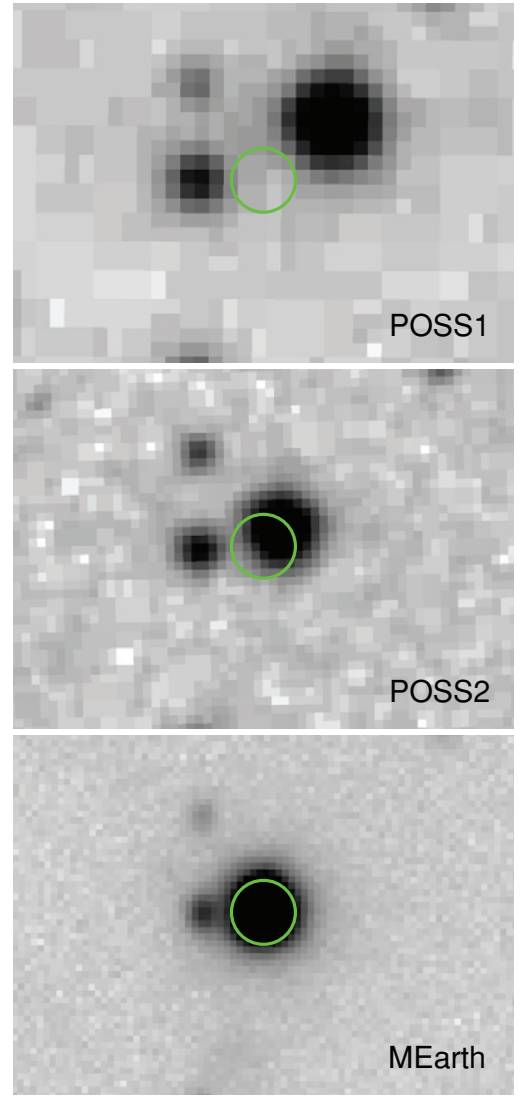

Figure 1. Images of GJ 3236 centered on its position as measured from the MEarth data. The circle shows the approximate position and size of the $5^{\prime \prime}$ (radius) photometric aperture used to derive our light curves. Data are from the first and second epoch Palomar sky surveys as provided by the Digitized Sky Survey (top and center panels) and the MEarth stacked master image (bottom panel). The approximate epochs of the images are 1954.1 (POSS-1), 1992.7 (POSS-2), and 2008.8 (MEarth). All three panels have the same center, scale, and alignment on-sky, with north up and east to the left, covering $1^{\prime}$ in the horizontal direction.

(A color version of this figure is available in the online journal.)

series of three images centered on the position of the photometric aperture used for GJ 3236 in the MEarth images, demonstrating that there are no such objects. If any additional third light is indeed present, it must therefore be comoving with GJ 3236, meaning it is highly likely to be physically associated, given the extremely small probability of such a chance alignment.

The photometry was calibrated using observations of standard star fields from Landolt (1992), taken automatically each night by the MEarth observation scheduling software. By fitting all the standard star observations from several photometric nights, we derived the following color equation to convert between the MEarth bandpass and the standard Johnson-Cousins system:

$$
\text { MEarth }=I_{\mathrm{C}}-0.18\left(V_{\mathrm{J}}-I_{\mathrm{C}}\right) .
$$

The full MEarth light curve is reproduced in Table 1.

\subsection{FLWO $1.2 \mathrm{~m} \mathrm{V-band} \mathrm{Follow-up} \mathrm{Photometry}$}

Observations centered around the primary eclipse of UT 2008 September 25 and the secondary eclipse of UT 2008 September 27 were obtained using the KeplerCam instrument on the Fred Lawrence Whipple Observatory (FLWO) $1.2 \mathrm{~m}$ telescope. We used the standard binning $2 \times 2$ readout mode, since the plate scale of 0!'34 per unbinned pixel significantly oversamples the 
Table 1

MEarth Light Curve of GJ 3236

\begin{tabular}{cccccccc}
\hline \hline HJD & MEarth & Error $^{\mathrm{a}}$ & $\Delta m^{\mathrm{b}}$ & FWHM (pixel) $^{\mathrm{c}}$ & Airmass $^{*} x\left(\right.$ pixel $^{\mathrm{d}}$ & $y($ pixel) \\
\hline 2454741.691783 & 11.1329 & 0.0036 & -0.278 & 7.24 & 1.94119 & 1029.66 & 1048.57 \\
2454741.704376 & 11.1151 & 0.0035 & -0.337 & 7.18 & 1.85590 & 1030.44 & 1049.71 \\
2454741.716182 & 11.1159 & 0.0035 & -0.223 & 6.78 & 1.78328 & 1030.33 & 1048.66 \\
2454741.727410 & 11.1127 & 0.0035 & -0.209 & 6.81 & 1.72032 & 1030.19 & 1048.31 \\
2454741.738267 & 11.1180 & 0.0035 & -0.226 & 6.95 & 1.66467 & 1030.41 & 1048.00 \\
\hline
\end{tabular}

Notes.

a Estimated using a standard CCD noise model, including contributions from Poisson noise in the stellar counts, sky noise, readout noise, and errors in the sky background estimation.

b Correction to the frame magnitude zero point applied in the differential photometry procedure. More negative numbers indicate greater losses.

${ }^{c}$ Median FWHM of the stellar images on the frame. The plate scale was 0.757 pixel $^{-1}$

d $x$ and $y$ pixel coordinates of GJ 3236 on the CCD image, derived using a standard intensity-weighted moments analysis.

(This table is available in its entirety in a machine-readable form in the online journal. A portion is shown here for guidance regarding its form and content.)

Table 2

$V$-band Light Curve of GJ 3236

\begin{tabular}{cccccccc}
\hline \hline HJD & $V$ & Error $^{\mathrm{a}}$ & $\Delta m^{\mathrm{b}}$ & FWHM (pixel) $^{\mathrm{c}}$ & Airmass & $x$ (pixel) $^{\mathrm{d}}$ & $y$ (pixel) $^{\mathrm{d}}$ \\
\hline 2454734.882776 & 14.2717 & 0.0030 & -0.137 & 6.29 & 1.30113 & 953.69 & 1372.35 \\
2454734.884327 & 14.2676 & 0.0029 & -0.094 & 6.08 & 1.29916 & 953.59 & 1372.41 \\
2454734.885866 & 14.2672 & 0.0029 & -0.068 & 5.92 & 1.29726 & 953.64 & 1372.36 \\
2454734.887406 & 14.2698 & 0.0029 & -0.112 & 6.14 & 1.29541 & 953.69 & 1372.47 \\
2454734.888957 & 14.2696 & 0.0029 & -0.093 & 6.05 & 1.29360 & 953.66 & 1372.63 \\
\hline
\end{tabular}

Notes.

${ }^{a}$ Estimated using a standard CCD noise model, including contributions from Poisson noise in the stellar counts, sky noise, readout noise, and errors in the sky background estimation.

${ }^{\mathrm{b}}$ Correction to the frame magnitude zero point applied in the differential photometry procedure. More negative numbers indicate greater losses.

${ }^{c}$ Median FWHM of the stellar images on the frame. The plate scale was $0{ }^{\prime} .67 \mathrm{pixel}^{-1}$.

${ }^{\mathrm{d}} x$ and $y$ pixel coordinates of GJ 3236 on the CCD image, derived using a standard intensity-weighted moments analysis.

(This table is available in its entirety in a machine-readable form in the online journal. A portion is shown here for guidance regarding its form and content.)

typical seeing at FLWO. The resulting plate scale was 0.'67 per summed pixel. We used the $V$ filter and an exposure time of $120 \mathrm{~s}$. We also used observations of standard star fields from Landolt (1992) taken on UT 2008 September 28 to calibrate the KeplerCam photometry onto the standard Johnson-Cousins system. A total of 245 observations were taken, where possible starting $2 \mathrm{hr}$ before first contact and finishing $2 \mathrm{hr}$ after last contact to sample the out-of-eclipse portions of the light curve and thus allow the eclipse depths to be properly measured. The end of the primary eclipse was missed due to twilight.

These photometric data were reduced using the same pipeline as described in Section 2.1. We used an aperture radius of 5 binned pixels, corresponding to 3".4 on-sky (recalling from Section 2.1 that the size of this aperture is important due to the nearby star). The difference in magnitude between GJ 3236 and the fainter star is only $2.3 \mathrm{mag}$ in $V$ band, which leads to approximately a $0.6 \%$ error in the measured fluxes of GJ 3236, and thus a similar error in the measured eclipse depths. Since the point-spread functions on our images are not very well behaved, it will be challenging to reduce this using PSF-fitting photometry, and it is still substantially smaller than the other sources of error in the present light curve models, so we have not pursued this issue further.

The full $V$-band light curve is reproduced in Table 2.

\subsection{PAIRITEL J-band Follow-up Photometry}

Observations in the near-infrared $J$ band were obtained using the robotic Peters automated infrared imaging telescope (PAIRITEL) from UT 2008 February 17 to UT 2008 March 3 (inclusive). We scheduled observations around the times of primary and secondary eclipses, obtaining 457 data points spread over 10 nights. Exposure times were $7.8 \mathrm{~s}$. The observations were automatically scheduled, collected, and reduced by the fully robotic PAIRITEL observing system (Bloom et al. 2006; Blake et al. 2005). We then produced differential photometry using a set of comparison stars chosen from the Two Micron All Sky Survey (2MASS) catalog. We estimate a photometric error of approximately $2 \%$ per data point from the scatter of the out-of-eclipse measurements. The full $J$-band light curve is reproduced in Table 3.

\subsection{Spectroscopy}

Spectroscopic observations were obtained using the TRES fiber-fed échelle spectrograph on the FLWO $1.5 \mathrm{~m}$ Tillinghast reflector. We used the medium fiber ( 2 ". 3 projected diameter) throughout, yielding a resolving power of $R \simeq 48,000$.

Table 4 summarizes these measurements, including the radial velocities of both components of the binary derived therefrom. 
Table 3

J-band Light Curve of GJ 3236

\begin{tabular}{ccc}
\hline \hline HJD & $J$ & Error $^{\mathrm{a}}$ \\
\hline 2454513.6413 & 10.015 & 0.020 \\
2454513.6421 & 10.040 & 0.020 \\
2454513.6430 & 10.052 & 0.020 \\
2454513.6438 & 10.078 & 0.020 \\
2454513.6447 & 10.042 & 0.020 \\
\hline
\end{tabular}

Note.

${ }^{a}$ Equal observational errors of 0.02 mag were assumed on each data point

(This table is available in its entirety in a machinereadable form in the online journal. A portion is shown here for guidance regarding its form and content.)

The TRES instrument is extremely stable, so we acquired ThAr wavelength calibration exposures before or after the target exposures, rather than using the simultaneous calibration fiber, which can lead to contamination of the target spectrum in the red-most orders from the very strong Ar lines in the ThAr lamp spectrum. A second fiber was placed on sky, but this was not used due to a problem causing extremely poor throughput in the TRES sky fiber, which has since been resolved.

Spectra were reduced using standard procedures in $\operatorname{IRAF}^{5}$ (Tody 1993) from the ECHELLE package to extract the spectra from the target fiber and sky fiber simultaneously. The TRES CCD is read out using two amplifiers, which necessitates combining them before tracing and extraction. This was done using the MSCRED package in IRAF, applying a multiplicative gain correction to equalize the difference in gain between the two readout electronics chains. We then divided by a normalized flat-field exposure to correct for fringing in the red-most regions of the spectrum, which has a significant effect on the orders we used for the radial velocity analysis.

At the time of our observations, the TRES detector suffered from a very high energetic particle hit rate, the source of which is under investigation. The particle hits were removed by mediancombining each set of multiple exposures for the flat fields, target and calibrations, and then using the statistics of the median frame to apply an upper envelope clip to the target frames themselves. This allowed us to avoid having to combine our target exposures to eliminate the particle hits, which would smear out the radial velocity variations slightly as a result of the extremely short orbital period of GJ 3236.

The stability of the TRES instrument allowed us to define the aperture trace for each fiber using the high signal-to-noise flat fields, which were then used to extract the target and calibration spectra, employing the "optimal" (in a least squares sense) weighting scheme of Hewett et al. (1985). The spectra were wavelength-calibrated using the standard ECIDENTIFY procedure, which employs the (known) dispersion relation of the échelle to solve for a wavelength solution over all orders of the spectrum simultaneously. This is necessary due to the relative paucity of sufficiently intense lines from the ThAr lamp in each order of the spectrum at wavelengths $>7500 \AA$.

\footnotetext{
5 IRAF is distributed by the National Optical Astronomy Observatories, which is operated by the Association of Universities for Research in Astronomy, Inc., under cooperative agreement with the National Science
} Foundation.

\subsection{Summary of System Properties}

Table 5 summarizes the known system properties, from the literature (principally the proper motion survey of Lépine \& Shara 2005 used to select the target stars for the MEarth survey) and our own $V$ and MEarth photometry, converted to the standard Johnson-Cousins system.

The average color of $V_{\mathrm{J}}-I_{\mathrm{C}}=2.73 \pm 0.07$ indicates an average spectral type of approximately M4 using the color- to spectral-type relation of Leggett (1992) for young Galactic disk stars.

\section{INITIAL LIGHT CURVE ANALYSIS (ЕВOP)}

For detached eclipsing binaries with circular orbits, the radial velocity (RV) and light curve models are largely independent. We therefore carried out a preliminary analysis of the available light curves before starting to obtain spectroscopic observations. The principal purpose of doing this was to determine an extremely precise orbital period to better target the radial velocity observations, but we can also constrain the system eccentricity by using the phase of the secondary eclipses relative to the primary eclipses (related to $e \cos \omega$ ). A simple geometric argument can then be used to obtain a limit on the eccentricity itself, assuming no a priori knowledge of $\omega$.

We used JKTEBOP (Southworth et al. 2004a, 2004b), a modified version of ЕВOP (Eclipsing Binary Orbit Program; Popper \& Etzel 1981; Etzel 1981), to obtain these parameters by fitting the MEarth light curve. The program was modified to fit simultaneously for the EB model, and a simple form for the synchronized out-of-eclipse modulations, assuming they can be approximated by a truncated Fourier series:

$$
m(t)=a_{1} \sin (\Omega t)+b_{1} \cos (\Omega t)+a_{2} \sin (2 \Omega t)+b_{2} \cos (2 \Omega t),
$$

where $\Omega=2 \pi / P$, and $P$ is the orbital period of the binary. The normalization term is omitted since this is already taken into account by the standard EBOP model. The revised code yields a fit with very small residuals for the present system, and the use of the form in Equation (2) keeps the number of parameters required to describe the modulations to a minimum compared to a full spot model. Doing so will not necessarily reproduce the correct eclipse depths for a given set of physical parameters (or vice versa), but should be adequate for deriving an accurate system ephemeris. We relax this assumption in Section 5, where we adopt a full spot model for determining the geometric and radiative parameters of the system.

The following parameters were allowed to vary in the fit: the sum of the radii divided by the semimajor axis, $r_{1}+r_{2}$ (where $r_{j}=R_{j} / a, R_{j}$ is the stellar radius of star $j$, and $a$ is the semimajor axis), orbital inclination $i, e \cos \omega$, central surface brightness ratio $J$ (essentially equal to the ratio of the primary and secondary eclipse depths), orbital period $P$, orbital phase zero-point $t_{0}$, light curve normalization, and the parameters $a_{1}$, $a_{2}, b_{1}$, and $b_{2}$ describing the out-of-eclipse modulations. Singleband light curves showing grazing eclipses constrain the ratio of the radii only very weakly (or equivalently, the luminosity ratio), so these parameters and the mass ratio (used for computing the oblateness of the stars and the reflection effect) were fixed at 1.0 for the initial analysis. The results for the orbital parameters were found not to change significantly upon revising these to the measured values from the spectroscopy once they were available.

Our light curves are not of sufficient quality to fit for the stellar limb darkening, so we assumed a square root limb-darkening 
Table 4

Barycentric Radial Velocity Measurements of GJ 3236

\begin{tabular}{|c|c|c|c|c|c|c|}
\hline HJD & \multicolumn{2}{|c|}{ Absorption (Barnard's Star) ${ }^{\mathrm{a}}$} & \multicolumn{2}{|c|}{ Emission (GJ 856A) } & \multicolumn{2}{|c|}{ Emission (GJ 3379) } \\
\hline 2454755.9072 & & & -44.23 & 86.66 & -42.91 & 87.65 \\
\hline 2454755.9154 & & & -49.99 & 92.02 & -48.28 & 91.13 \\
\hline 2454755.9241 & & & -53.77 & 100.02 & -52.82 & 99.68 \\
\hline 2454757.8115 & 55.29 & -37.60 & 55.70 & -44.31 & 56.17 & -42.63 \\
\hline 2454757.8352 & 66.54 & -66.09 & 70.44 & -63.70 & 71.87 & -62.77 \\
\hline 2454756.8324 & & & -66.67 & 118.68 & -66.39 & 119.91 \\
\hline 2454756.8406 & & & -64.81 & 114.28 & -63.84 & 116.20 \\
\hline 2454756.8483 & & & -63.87 & 114.44 & -62.96 & 117.50 \\
\hline 2454729.8825 & & & -50.95 & 96.13 & -49.89 & 96.98 \\
\hline 2454729.9057 & -38.67 & 74.80 & -40.44 & 77.21 & -39.52 & 79.13 \\
\hline 2454759.9137 & & & -59.96 & 119.56 & -58.57 & 123.32 \\
\hline 2454759.9252 & & & -61.53 & 104.53 & -55.20 & 102.53 \\
\hline 2454759.9513 & & & -57.89 & 106.49 & -56.91 & 107.42 \\
\hline 2454758.8510 & 51.55 & -31.67 & 48.34 & -37.76 & 48.98 & -35.47 \\
\hline 2454758.8587 & 48.84 & -26.92 & 44.02 & -32.35 & 44.54 & -30.38 \\
\hline 2454758.8668 & 48.57 & -21.32 & 39.19 & -21.43 & 40.09 & -18.55 \\
\hline 2454758.8745 & 38.38 & -17.90 & 33.12 & -16.09 & 34.85 & -12.88 \\
\hline 2454730.9731 & 90.88 & -101.58 & 100.49 & -101.59 & 102.23 & -100.68 \\
\hline 2454730.9768 & 79.42 & -110.01 & 101.24 & -96.71 & 102.05 & -96.08 \\
\hline 2454732.0024 & & & -45.76 & 91.06 & -44.87 & 92.26 \\
\hline
\end{tabular}

Note.

${ }^{\text {a }}$ Radial velocities are reported only for epochs where there was a sufficient signal-to-noise ratio to obtain a usable cross-correlation. This was satisfied for all the epochs in the emission line analysis but only for 21 epochs in the absorption line analysis, due to the higher signal-to-noise ratio of the strong $\mathrm{H} \alpha$ lines compared to the continuum.

law with coefficients appropriate to the $I$ (Cousins) passband from Claret (2000) using the PHOENIX model atmospheres. Given the lack of a spectroscopic constraint on the effective temperature, surface gravity, and metallicity for either star, we have instead assumed solar metallicity, and derived temperatures and surface gravities of $T_{1}=3280 \mathrm{~K}$ and $\log g_{1}=4.9$, and for the secondary, $T_{2}=3200 \mathrm{~K}$ and $\log g_{2}=5.0$ by iterating the modeling process. The assumed temperatures were derived using the masses from the combined orbital and light curve solution, interpolating between the compilation of values for field stars by Leggett (1992). See also Section 6 where these temperatures are verified using the final model. A gravity darkening exponent of $\beta=0.32$, a value appropriate for stars with convective envelopes (Lucy 1967), was also assumed, and the option in JKTEBOP to calculate the reflection effect was used rather than fitting for it.

We report only the orbital parameters $\left(P, t_{0}\right.$, and $\left.e \cos \omega\right)$ from the EBOP analysis in Table 6, and have adopted these for the remainder of this work.

The parameter uncertainties were derived using a Monte Carlo algorithm built-in to JKTEBOP (Southworth et al. 2005). Briefly, this algorithm uses the best fit to generate a synthetic light curve, injecting Gaussian noise with amplitude determined by the observational errors (which were scaled such that the reduced $\chi^{2}$ of the fit was equal to unity) to produce a simulated light curve, which is then fitted to determine a new set of parameters. The parameter uncertainties can be estimated using the distribution of the parameters from a large number of realizations (here, 10,000) of this process. See also Southworth et al. (2004a, 2004b) for more details.

Times of minimum light derived for the 11 well sampled eclipses in our $V$ and MEarth observations are reported in Table 7. These were estimated using the method of Kwee \& van Woerden (1956) over a \pm 0.03 region in normalized orbital phase around each eclipse. We subtracted the truncated Fourier series in Equation (2) from the light curves before applying this analysis to reduce the effect of any systematic errors arising from the out-of-eclipse modulation.

\section{RADIAL VELOCITY ANALYSIS}

Radial velocities were obtained using the two-dimensional cross-correlation algorithm TODCOR (Zucker \& Mazeh 1994), 
Table 5

Summary of the Photometric and Astrometric Properties of the GJ 3236 System

\begin{tabular}{lc}
\hline \hline Parameter & Value \\
\hline$\alpha_{2000}{ }^{\mathrm{a}, \mathrm{b}}$ & $03^{\mathrm{h}} 37^{\mathrm{m}} 14^{\mathrm{s}} .08$ \\
$\delta_{2000}{ }^{\mathrm{a}, \mathrm{b}}$ & $+69^{\circ} 10^{\prime} 49^{\prime \prime} .8$ \\
$\mu_{\alpha} \cos \delta^{\mathrm{b}}$ & $0^{\prime \prime} 142 \mathrm{yr}^{-1}$ \\
$\mu_{\delta}^{\mathrm{b}}$ & $-0^{\prime \prime} 132 \mathrm{yr}^{-1}$ \\
$\mathrm{MEarth}^{\mathrm{c}}$ & $11.05 \pm 0.05$ \\
$V_{\mathrm{J}}^{\mathrm{c}}$ & $14.28 \pm 0.05$ \\
$I_{\mathrm{C}}^{\mathrm{c}}$ & $11.55 \pm 0.05$ \\
$J_{2 \mathrm{MASS}}{ }^{\mathrm{d}}$ & $9.806 \pm 0.023$ \\
$H_{2 \mathrm{MASS}}{ }^{\mathrm{d}}$ & $9.198 \pm 0.028$ \\
$K_{2 \mathrm{MASS}}{ }^{\mathrm{d}}$ & $8.967 \pm 0.022$ \\
\hline
\end{tabular}

Notes.

${ }^{a}$ Equinox J2000.0, epoch 2000.0.

b From Lépine \& Shara (2005).

${ }^{c}$ Median of the measured out-of-eclipse fluxes from our light curves. We estimate that the observational errors are dominated by the uncertainty in the photometric calibration, which is approximately $0.05 \mathrm{mag}$ for both passbands. $I_{\mathrm{C}}$ was computed from the measured MEarth and $V_{\mathrm{J}}$ magnitudes using Equation (1).

${ }^{d}$ We quote the combined uncertainties from the 2MASS catalog, noting that the intrinsic variability of our target means in practice that these are underestimates.

which uses templates matched to each component of a spectroscopic binary to simultaneously derive the velocities of both stars, and importantly for the present application, an estimate of their luminosity ratio $(\alpha)$ in the spectral bandpass.

We performed both a standard absorption line based crosscorrelation analysis and an emission line analysis using the strong $\mathrm{H} \alpha$ emission observed in both components of GJ 3236. The signal-to-noise ratio of the latter is substantially higher in our data, but we caution that the source of the $\mathrm{H} \alpha$ emission in our target stars is not well understood. The emission line analysis results could suffer systematic errors depending on the relative velocities of the regions of the chromosphere emitting the $\mathrm{H} \alpha$ and the stellar photospheres, and this is exacerbated in the present system because it is highly likely that the stellar spin and binary orbit are synchronized, both from the light curves and expectations from tidal theory (Zahn 1977). We therefore conservatively adopt the results from the absorption line analysis despite the larger errors.

The absorption line analysis used as a template spectrum a single epoch observation of Barnard's star (Barnard 1916; also known as GJ 699) taken on UT 2008 October 20. We used a wavelength range of $8700-8850 \AA$ in order 50 of the spectrum to derive the velocities, since this region contains a number of reasonably strong metallic lines in $\mathrm{M}$ dwarfs, and is free of telluric absorption lines. The Ca II infrared triplet at 8498 , 8542 , and $8662 \AA$ was deliberately avoided since the absorption in these lines is saturated, and they are therefore intrinsically very broad, and emission cores are often observed in these lines in active stars, which would severely complicate the use of an inactive field star as a template. We assumed a barycentric radial velocity of $-110.13 \mathrm{~km} \mathrm{~s}^{-1}$ for Barnard's star, derived from presently unpublished CfA Digital Speedometer measurements spanning 17 years.

For the emission line analysis, we used as templates spectra of two M4 dwarfs that were found to display $\mathrm{H} \alpha$ emission, GJ 856A, and GJ 3379, observed as part of another program
Table 6

Derived Orbital Parameters for the GJ 3236 System

\begin{tabular}{|c|c|}
\hline Parameter & Value $^{\mathrm{a}}$ \\
\hline$P$ & $0.7712600 \pm 0.0000023$ days \\
\hline$t_{0}$ & $2454734.99586 \pm 0.00012 \mathrm{HJD}^{\mathrm{b}}$ \\
\hline$e \cos \omega$ & $0.00008 \pm 0.00020$ \\
\hline$e$ & $<0.0022(95 \% \mathrm{CL})^{\mathrm{c}}$ \\
\hline & $<0.0112(99 \% \mathrm{CL})^{\mathrm{c}}$ \\
\hline$\alpha$ & $0.60 \pm 0.04$ \\
\hline \multicolumn{2}{|c|}{ Absorption line solution (adopted) } \\
\hline$\gamma$ & $10.06 \pm 0.94 \mathrm{~km} \mathrm{~s}^{-1}$ \\
\hline$K_{1}$ & $85.6 \pm 2.1 \mathrm{~km} \mathrm{~s}^{-1}$ \\
\hline$K_{2}$ & $114.8 \pm 1.9 \mathrm{~km} \mathrm{~s}^{-1}$ \\
\hline$q$ & $0.746 \pm 0.023$ \\
\hline$a \sin i$ & $3.053 \pm 0.044 R_{\odot}$ \\
\hline$M_{1} \sin ^{3} i$ & $0.368 \pm 0.015 M_{\odot}$ \\
\hline$M_{2} \sin ^{3} i$ & $0.275 \pm 0.014 M_{\odot}$ \\
\hline$\sigma_{1}$ & $6.3 \mathrm{~km} \mathrm{~s}^{-1 \mathrm{~d}}$ \\
\hline$\sigma_{2}$ & $5.6 \mathrm{~km} \mathrm{~s}^{-1}$ \\
\hline \multicolumn{2}{|c|}{ H $\alpha$ emission line solution $^{\mathrm{e}}$ : GJ 856A } \\
\hline$\gamma$ & $12.87 \pm 0.19 \mathrm{~km} \mathrm{~s}^{-1}$ \\
\hline$K_{1}$ & $88.48 \pm 0.33 \mathrm{~km} \mathrm{~s}^{-1}$ \\
\hline$K_{2}$ & $114.71 \pm 0.49 \mathrm{~km} \mathrm{~s}^{-1}$ \\
\hline$q$ & $0.7713 \pm 0.0045$ \\
\hline$a \sin i$ & $3.0962 \pm 0.0091 R_{\odot}$ \\
\hline$M_{1} \sin ^{3} i$ & $0.3786 \pm 0.0037 M_{\odot}$ \\
\hline$M_{2} \sin ^{3} i$ & $0.2919 \pm 0.0025 M_{\odot}$ \\
\hline$\sigma_{1}$ & $1.3 \mathrm{~km} \mathrm{~s}^{-1}$ \\
\hline$\sigma_{2}$ & $1.9 \mathrm{~km} \mathrm{~s}^{-1}$ \\
\hline \multicolumn{2}{|c|}{ H $\alpha$ emission line solution $^{\mathrm{e}}$ : GJ 3379} \\
\hline$\gamma$ & $13.98 \pm 0.20 \mathrm{~km} \mathrm{~s}^{-1}$ \\
\hline$K_{1}$ & $88.65 \pm 0.34 \mathrm{~km} \mathrm{~s}^{-1}$ \\
\hline$K_{2}$ & $114.87 \pm 0.52 \mathrm{~km} \mathrm{~s}^{-1}$ \\
\hline$q$ & $0.7717 \pm 0.0047$ \\
\hline$a \sin i$ & $3.1011 \pm 0.0096 R_{\odot}$ \\
\hline$M_{1} \sin ^{3} i$ & $0.3802 \pm 0.0039 M_{\odot}$ \\
\hline$M_{2} \sin ^{3} i$ & $0.2934 \pm 0.0026 M_{\odot}$ \\
\hline$\sigma_{1}$ & $1.3 \mathrm{~km} \mathrm{~s}^{-1}$ \\
\hline$\sigma_{2}$ & $2.0 \mathrm{~km} \mathrm{~s}^{-1}$ \\
\hline
\end{tabular}

Notes.

${ }^{a}$ We report $68.3 \%$ confidence intervals, with error bars symmetrized by adopting the mean of the $15.85 \%$ and $85.15 \%$ levels as the central value.

${ }^{\mathrm{b}}$ Ephemeris zero point, chosen to correspond to the epoch of the first primary eclipse in the $V$-band data from Section 2.2.

c Derived assuming only the measured $e \cos \omega$ and a uniform distribution in $\omega$, for $95 \%$ and $99 \%$ confidence levels. These confidence levels are in fact lower limits, since there were no noticeable differences in the eclipse durations, or deviations from a circular orbit in radial velocity. $e=0$ was assumed henceforth for the radial velocity modeling.

$\mathrm{d}$ rms of the residuals after subtracting the Keplerian orbit model from the data. These are representative of the typical uncertainty on each RV point.

e We re-emphasize that the $\mathrm{H} \alpha$ radial velocities, despite having smaller random errors than the absorption line velocities, may have an unknown and potentially significant systematic error, due to the uncertainty in the source of the $\mathrm{H} \alpha$ emission line, and whether it tracks the stellar photosphere. We therefore conservatively adopt the absorption line solution for the remainder of the present publication.

using the same instrument. GJ 856A was observed on UT 2008 October 17 and GJ 3379 was observed on UT 2008 October 19. We used a wavelength range of 6520-6645 $\AA$ in order 37 
Table 7

Measured Times of Minimum Light for GJ 3236

\begin{tabular}{crrc}
\hline \hline HJD & \multicolumn{1}{c}{$(O-C)(\mathrm{s})$} & Cycle $^{\mathrm{a}}$ & Band \\
\hline 2454734.995569 & $-32.5 \pm 52.0$ & 0.0 & $V$ \\
2454736.923700 & $-34.5 \pm 26.7$ & 2.5 & $V$ \\
2454741.937128 & $-7.8 \pm 17.0$ & 9.0 & MEarth \\
2454742.708283 & $-19.6 \pm 18.9$ & 10.0 & MEarth \\
2454745.793562 & $7.2 \pm 18.9$ & 14.0 & MEarth \\
2454762.761020 & $-22.0 \pm 25.2$ & 36.0 & MEarth \\
2454765.846327 & $7.9 \pm 78.0$ & 40.0 & MEarth \\
2454766.617531 & $1.7 \pm 13.7$ & 41.0 & MEarth \\
2454767.774152 & $-28.4 \pm 67.0$ & 42.5 & MEarth \\
2454770.859614 & $18.9 \pm 36.6$ & 46.5 & MEarth \\
2454771.630638 & $-7.6 \pm 38.6$ & 47.5 & MEarth \\
\hline
\end{tabular}

Note.

${ }^{\text {a }}$ Cycle number, counting from 0 at the primary eclipse at $t_{0}$, in units of the orbital period. Integer numbers correspond to primary eclipses.

for the emission line cross-correlations, noting that the crosscorrelations are dominated by the $\mathrm{H} \alpha$ lines.

Barycentric radial velocities of GJ 856A and GJ 3379 were determined by cross-correlation with Barnard's star, using the 8700-8850 $\AA$ region as for our absorption line analysis of GJ 3236.

Given that TODCOR provides a measure of the correlation between the observed and template spectra, it is possible to run cross-correlations against a series of templates and use the correlation value as an indicator of the template match. Both the effective temperature and the rotational velocity of the templates affect the solution, so it is vital to explore this parameter space. Due to the availability of a limited range of observed templates, the effective temperature could not be varied, but we were able to rotationally broaden the template spectra in order to create primary and secondary templates with $v_{\text {rot }} \in\{1,2,4,6,8,10,12,16,20,25,30,35,40\} \mathrm{km} \mathrm{s}^{-1}$. We used linear limb-darkening coefficients $u_{1}=0.5782$ and $u_{2}=0.5934$ from Claret (2004) for the SDSS $z$ passband (a close match to the effective wavelength of the spectroscopy), which correspond to our assumed temperatures for the primary and secondary (see Section 3).

We ran TODCOR on the grid of 169 template combinations and sought the maximum of the resulting correlation values to determine the most likely $v_{\text {rot }} \sin i$ values for the two components of the binary system. Barnard's star is a very slow rotator, so the rotation of the template is negligible given our spectral resolution. Our analysis indicates $v_{\text {rot, } 1} \sin i=25 \mathrm{~km} \mathrm{~s}^{-1}$, which is consistent with the expected value of $v_{\mathrm{rot}, 1} \sin i=24.6 \mathrm{~km} \mathrm{~s}^{-1}$ if the spin and orbit are synchronized. $v_{\mathrm{rot}, 2} \sin i$ was poorly constrained, with the maximum correlation occurring at $3 \mathrm{~km} \mathrm{~s}^{-1}$, whereas we would expect $19.5 \mathrm{~km} \mathrm{~s}^{-1}$ assuming synchronization. We have simply adopted the latter value, since the light curves and primary velocity indicate that the system is indeed synchronized. Using the templates with $v_{\mathrm{rot}, 1} \sin i=25 \mathrm{~km} \mathrm{~s}^{-1}$ and $v_{\text {rot, } 2} \sin i=20 \mathrm{~km} \mathrm{~s}^{-1}$, TODCOR indicates $\alpha=0.60 \pm 0.04$.

For the emission line analysis, we assumed the stellar rotation values from the absorption line analysis, rotationally broadening the observed spectra of GJ 856A and GJ 3379. The intrinsic rotational broadening in both of these templates is again negligible for our purposes. TODCOR gave a maximum correlation for $\alpha_{\mathrm{Ha}}=0.52 \pm 0.02$, which provides a measure of the relative $\mathrm{H} \alpha$ emission luminosities of the two stars.

Since the orbital period and time of primary eclipse are extremely well determined from the light curves, we held these values fixed in the final radial velocity orbital solution. We also assumed a circular orbit. Figure 2 shows the resulting radial velocity curves. The parameters derived from the radial velocity analysis are given in Table 6 . We assumed equal observational errors on each radial velocity point, scaled such that the reduced $\chi^{2}$ of the fit was equal to unity.

Radial velocities of the components of double-lined spectroscopic binaries are prone to a "peak pulling" effect as they approach the $\gamma$ velocity (at times of minimum separation of the two components in the spectrum). The TODCOR method can largely eliminate this, provided the template spectra match the target sufficiently well. In our experience, this effect is particularly sensitive to errors in the assumed rotational broadening: if the template contains too much or too little rotation, the velocities would be pushed toward or away from $\gamma$ to compensate. Examining the residuals of our solution in Figure 2 indicates little or no "peak pulling," which further justifies our assumed rotational velocities and the assumption of negligible rotation in the templates.

\section{LIGHT CURVE ANALYSIS (WILSON \& DEVINNEY CODE)}

In order to properly account for spots on the components of GJ 3236, we use the most recent version (WD2007) of the standard Wilson \& Devinney (1971) code to derive the geometric and radiative parameters of the system. This code incorporates a full physical model including spots and using model atmospheres.

WD normally uses the Kurucz (1979, 1993) model atmospheres to calculate the emergent flux from surface elements on each binary component. These models span an effective temperature range of 3500-50,000 K, and for temperatures below $3500 \mathrm{~K}$, WD interpolates between a black body of the appropriate temperature and the $3500 \mathrm{~K}$ model atmosphere. This is weighted in such a fashion as to produce a pure black body at $1500 \mathrm{~K}$, and for intermediate temperatures there is a smooth ramp toward a pure model atmosphere at $3500 \mathrm{~K}$. Molecular sources of opacity increasingly begin to dominate the optical spectra of $\mathrm{M}$ dwarfs in this temperature range, so a black body is clearly a poor approximation for the emergent flux. Since both components of GJ 3236 appear to be cooler than $3500 \mathrm{~K}$, we have modified our copy of WD to substitute PHOENIX NextGen model atmospheres (Hauschildt et al. 1999) in place of these interpolations for effective temperatures cooler than $3500 \mathrm{~K}$.

\subsection{Assumptions}

Our assumptions for the WD light curve model are detailed in Table 8. We have assumed zero orbital eccentricity, synchronized spin and orbit for both components of the binary, and no third light, which are justified in Sections 2.1 and 3.

The limited phase coverage and lack of repeat observations in our $V$-band photometry (leading to an uncertainty in any corrections for color-dependent "second-order" atmospheric extinction), and poor precision (large scatter) in the $J$-band photometry means in practice that these are not useful in aiding the determination of the geometric (and thus physical) parameters of the system. Our experiments with the WD model indicate that these provide minimal additional constraints on any of the parameters when compared to using the MEarth data alone. We have therefore elected to use only the MEarth data for fitting for the system parameters, and then 

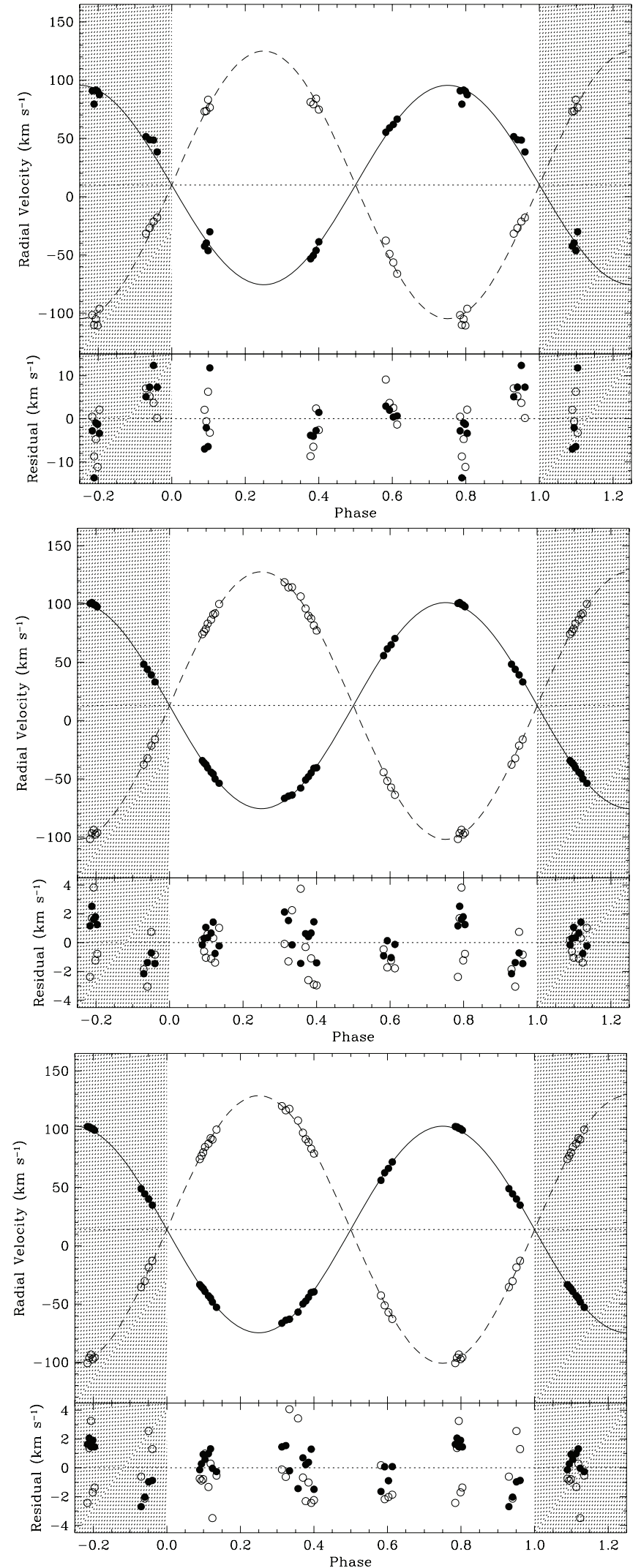

Figure 2. Phase-folded radial velocity curves for the GJ 3236 system. Shown in each panel are the radial velocities for the primary (filled symbols) and the secondary (open symbols), with the best-fitting Keplerian orbit overplotted using solid and dashed lines for the two components, respectively. The lower subpanels show the residuals after subtracting the model from the data Top: absorption line solution cross-correlating against Barnard's star; center: emission line solution using GJ 856A; bottom: emission line solution using GJ 3379 as the template. evaluated this model with respect to the other passbands as a check.

Doing so necessitates fixing one of the component effective temperatures, since the relative eclipse depths measured in a single passband merely determine the relative temperatures of the two components. We have therefore assumed $T_{1}=$ $3280 \mathrm{~K}$ following Section 3. In practice even given multiband photometry it is usually necessary to fix this parameter: although the color dependence of the eclipse depths does in principle provide information on the value of $T_{1}$, this is normally poorly constrained due to the difficulty of measuring differential colors to high precision.

In order to reproduce the out-of-eclipse variations seen in our optical light curves, at least two spots must be introduced into the model. The configuration of these spots affects the measured eclipse depths, depending on whether a spotted or unspotted part of the photosphere is eclipsed. This predominantly affects the derived ratio of surface brightnesses as measured by the ratio of depths of the primary and secondary eclipses (and thus the ratios of the component radii and effective temperatures inferred from this), and a combination of $\left(R_{1}+R_{2}\right) / a$ and the orbital inclination inferred from the total eclipse depth.

Since our $V$-band data do not cover the portions of the light curve out of eclipse, and the scatter in the $J$-band data is too large to usefully constrain the model, the spot modeling must be done using single-band light curve information. Spots are introduced in the WD code using a standard four-parameter model, where each spot is parameterized using the spot latitude $\theta_{s}$ and longitude $\phi_{s}$, radius $r_{s}$, and temperature contrast $T_{s} / T_{p}$. The latter quantity gives the ratio of effective temperatures of the spotted and unspotted photosphere. For a single-band light curve, the spot longitude is well constrained by the phase of the out-of-eclipse modulation, but the combination of $\theta_{s}, r_{s}$, and $T_{s} / T_{p}$ is largely degenerate (these quantities determine the amplitude and shape of the variation). Moreover, we can place each spot on either star. Although the data do rule out some of the possible spot locations, there are still a range of possibilities, which introduces a systematic error into the geometric parameters of the system. In principle, measuring the color dependence of the out-of-eclipse modulation would allow this degeneracy to be reduced.

Given the presently available data, we instead probe this systematic error by considering three characteristic spot configurations that are consistent with the light curve. These are (1) one close to polar spot on each star, with $\theta_{s}=60^{\circ}$, with both spots located in the same hemisphere as the chord traversed during the eclipse, resulting in the spot on the primary being eclipsed by the secondary during primary eclipse, and vice versa during secondary eclipse; (2) likewise, only with the spots located in the opposite hemisphere, resulting in no spots being eclipsed; and (3) a case intermediate between these, with a "symmetric" spot configuration of two spots on each star, at latitudes of $60^{\circ}$ with one located in each hemisphere. In each model, the spot latitudes and temperature ratios were held fixed, allowing only longitude and radius to vary, and in configuration (3) we enforced symmetry of the spot pattern about the equator, i.e., both spots on one star were forced to have the same longitudes and radii. This means that all three models have the same number of parameters (4).

\subsection{Model Fitting and Error Estimates}

To derive robust error estimates, including the correlations between parameters, we have implemented a Markov Chain Monte 
Table 8

Parameters Used in the WD Light Curve Model

\begin{tabular}{|c|c|c|c|}
\hline Parameter & WD Name & Value or "Varied" & Description \\
\hline Binary type & & Detached & \\
\hline$t_{0}$ & HJDO & 2454734.99586 (HJD) & Phase zero point ${ }^{\mathrm{a}}$ \\
\hline$P$ & PERIOD & 0.77126 days & Orbital period $^{\mathrm{a}}$ \\
\hline$d P / d t$ & DPDT & 0 & First derivative of period \\
\hline$a \sin i$ & $\mathrm{SMA} \times \sin i$ & $3.053 \pm 0.044 R_{\odot}$ & Projected semimajor axis ${ }^{\mathrm{a}, \mathrm{b}}$ \\
\hline$q$ & RM & $0.746 \pm 0.023$ & Mass ratio ${ }^{\mathrm{a}}$ \\
\hline$\gamma$ & VGA & $10.06 \pm 0.94 \mathrm{~km} \mathrm{~s}^{-1}$ & Systemic velocity $^{a}$ \\
\hline$e$ & $\mathrm{E}$ & 0 & Orbital eccentricity \\
\hline$F_{1}$ & $\mathrm{~F} 1$ & 1.0 & Primary rotation parameter \\
\hline \multirow[t]{2}{*}{$F_{2}$} & $\mathrm{~F} 2$ & 1.0 & Secondary rotation parameter \\
\hline & HLA & Varied & Light curve normalization \\
\hline$T_{1}$ & TAVH & $3280 \mathrm{~K}$ & Primary effective temperature \\
\hline$T_{2}$ & TAVC & Varied & Secondary effective temperature \\
\hline$\Omega_{1}$ & PHSV & Varied & Primary surface potential ${ }^{\mathrm{c}}$ \\
\hline$\Omega_{2}$ & PCSV & Varied & Secondary surface potential ${ }^{\mathrm{c}}$ \\
\hline$i$ & INCL & Varied & Orbital inclination \\
\hline$L_{3}$ & EL3 & 0 & Third light \\
\hline$A_{1}$ & ALB1 & 0.5 & Primary bolometric albedo ${ }^{\mathrm{d}}$ \\
\hline$A_{2}$ & ALB2 & 0.5 & Secondary bolometric albedo ${ }^{\mathrm{d}}$ \\
\hline$\beta_{1}$ & GR1 & 0.32 & Primary gravity darkening exponent ${ }^{\mathrm{d}}$ \\
\hline$\beta_{2}$ & GR2 & 0.32 & Secondary gravity darkening exponent ${ }^{\mathrm{d}}$ \\
\hline$\theta_{s, 1}$ & XLAT1 & $60^{\circ}$ & Spot 1 latitude $\mathrm{e}$ \\
\hline$\phi_{s, 1}$ & XLONG1 & Varied & Spot 1 longitude \\
\hline$r_{s, 1}$ & RADSP1 & Varied & Spot 1 radius \\
\hline$T_{s, 1} / T_{1}$ & TEMSP1 & 0.96 & Spot 1 temperature contrast \\
\hline$\theta_{s, 2}$ & XLAT2 & $60^{\circ}$ & Spot 2 latitude $^{\mathrm{e}}$ \\
\hline$\phi_{s, 2}$ & XLONG2 & Varied & Spot 2 longitude \\
\hline$r_{s, 2}$ & RADSP2 & Varied & Spot 2 radius \\
\hline$T_{s, 2} / T_{2}$ & TEMSP2 & 0.96 & Spot 2 temperature contrast \\
\hline$\alpha$ & & $0.60 \pm 0.04$ & Light ratio ${ }^{\mathrm{a}}$ \\
\hline
\end{tabular}

Notes.

a From Table 6.

${ }^{\mathrm{b}}$ Although the WD parameter SMA is the semimajor axis itself, we fixed $a \sin i$ in our MCMC procedure to the value from the radial velocities, calculating the required SMA value using $i$ (INCL) at each iteration.

c Modified Kopal (1959) potentials. These are roughly inversely proportional to stellar radii for this detached binary; for a clear description of their definition, we refer the reader to the documentation for the new WD graphical user interface PHOEBE (Prša \& Zwitter 2005).

d Values appropriate for convective atmospheres.

e See the text.

Carlo (MCMC) algorithm around the WD light curve model. Following Ford (2005), we used the Metropolis-Hastings algorithm (Metropolis et al. 1953; Hastings 1970) to estimate the a posteriori joint probability distribution of the fitted model parameters. The fitting statistic was the traditional $\chi^{2}$, where the observational error estimates were scaled such that each light curve's reduced $\chi^{2}$ was equal to unity.

We briefly summarize the Metropolis-Hastings algorithm here. Starting from an initial point in parameter space, the algorithm takes the most recent set of parameters and perturbs one or more parameters by a random Gaussian deviate. If the perturbed parameter set has a lower $\chi^{2}$ than its progenitor, it is accepted as a new point in the chain. If it has a larger $\chi^{2}$, it is accepted with a probability $\exp \left(-\Delta \chi^{2} / 2\right)$. If it is not accepted, the original point is repeated in the chain. The size of the perturbations were adjusted so that $20 \%-30 \%$ of the proposed points were accepted.

We included the spectroscopic light ratio between the primary and secondary components as an observation in our $\chi^{2}$ statistic, which in practice was implemented by adding an extra contribution to $\chi^{2}$ of the form

$$
\Delta \chi_{\text {prior }}^{2}=\left[\frac{\alpha_{\text {measured }}-\alpha_{\mathrm{WD}}}{\sigma_{\alpha}}\right]^{2},
$$

where $\alpha_{\text {measured }}$ and $\sigma_{\alpha}$ are the observed light ratio and its error, taken from Table 8 , and $\alpha_{\mathrm{WD}}$ is the value calculated from the WD model. This is necessary for systems with nearcircular orbits and grazing eclipses because the light curves only very weakly constrain this quantity (or equivalently, the ratio of the component radii). Uninformative (uniform) priors were assumed on all other parameters.

The final parameters and error estimates were determined by taking the $68.3 \%$ confidence interval (15.85 and 84.15 percentiles, corresponding to \pm 1 standard deviation for a Gaussian distribution) of the samples generated by our MCMC procedure. The chains were run until they contained at least $10^{6}$ samples, and we discarded the first $10 \%$ of the points in each 
Table 9

Derived Geometric, Radiative, and Physical Parameters of the GJ 3236 System

\begin{tabular}{|c|c|c|c|}
\hline \multirow[t]{2}{*}{ Parameter } & \multicolumn{3}{|c|}{ Value $^{\mathrm{a}}$} \\
\hline & Model 1 & Model 2 & Model 3 \\
\hline HLA & $0.0002759 \pm 0.0000073$ & $0.0003037 \pm 0.0000014$ & $0.0002915 \pm 0.0000066$ \\
\hline$T_{2} / T_{1}$ & $0.9801 \pm 0.0016$ & $0.97914 \pm 0.00085$ & $0.9774 \pm 0.0014$ \\
\hline$\Omega_{1}$ & $9.01 \pm 0.12$ & $8.781 \pm 0.030$ & $8.794 \pm 0.098$ \\
\hline$\Omega_{2}$ & $8.39 \pm 0.14$ & $9.236 \pm 0.041$ & $8.806 \pm 0.156$ \\
\hline$i$ & $82.805 \pm 0.041$ & $83.721 \pm 0.032$ & $83.154 \pm 0.047$ \\
\hline$\phi_{s, 1}$ & $-17.2 \pm 1.5$ & $-14.0 \pm 1.4$ & $-21.6 \pm 2.0$ \\
\hline$r_{s, 1}$ & $36.5 \pm 1.1$ & $43.83 \pm 0.41$ & $25.90 \pm 0.42$ \\
\hline$\phi_{s, 2}$ & $17.5 \pm 1.4$ & $20.4 \pm 1.2$ & $22.0 \pm 1.1$ \\
\hline$r_{s, 2}$ & $46.7 \pm 1.8$ & $59.98 \pm 0.93$ & $34.54 \pm 0.65$ \\
\hline$\left(R_{1}+R_{2}\right) / a$ & $0.22397 \pm 0.00082$ & $0.21670 \pm 0.00073$ & $0.22137 \pm 0.00085$ \\
\hline$R_{2} / R_{1}$ & $0.850 \pm 0.028$ & $0.7380 \pm 0.0048$ & $0.783 \pm 0.024$ \\
\hline$M_{1}$ & $0.377 \pm 0.016 M_{\odot}$ & $0.375 \pm 0.016 M_{\odot}$ & $0.376 \pm 0.017 M_{\odot}$ \\
\hline$M_{2}$ & $0.282 \pm 0.015 M_{\odot}$ & $0.280 \pm 0.015 M_{\odot}$ & $0.281 \pm 0.015 M_{\odot}$ \\
\hline$R_{1}$ & $0.3729 \pm 0.0078 R_{\odot}$ & $0.3829 \pm 0.0057 R_{\odot}$ & $0.3828 \pm 0.0072 R_{\odot}$ \\
\hline$R_{2}$ & $0.3167 \pm 0.0075 R_{\odot}$ & $0.2828 \pm 0.0043 R_{\odot}$ & $0.2992 \pm 0.0075 R_{\odot}$ \\
\hline$a$ & $3.077 \pm 0.044$ & $3.071 \pm 0.044$ & $3.075 \pm 0.044 R_{\odot}$ \\
\hline $\log g_{1}$ & $4.872 \pm 0.015$ & $4.8456 \pm 0.0081$ & $4.850 \pm 0.014$ \\
\hline $\log g_{2}$ & $4.886 \pm 0.019$ & $4.9819 \pm 0.0115$ & $4.935 \pm 0.019$ \\
\hline$v_{\text {rot }, 1}$ & $24.44 \pm 0.52 \mathrm{~km} \mathrm{~s}^{-1}$ & $25.12 \pm 0.37 \mathrm{~km} \mathrm{~s}^{-1}$ & $25.05 \pm 0.50 \mathrm{~km} \mathrm{~s}^{-1}$ \\
\hline$v_{\text {rot }, 2}$ & $20.77 \pm 0.47 \mathrm{~km} \mathrm{~s}^{-1}$ & $18.54 \pm 0.27 \mathrm{~km} \mathrm{~s}^{-1}$ & $19.60 \pm 0.44 \mathrm{~km} \mathrm{~s}^{-1}$ \\
\hline$N_{\text {samp }}$ & $1.61 \times 10^{6}$ & $2.35 \times 10^{5}$ & $1.37 \times 10^{6}$ \\
\hline$\sigma_{\text {MEarth }}{ }^{b}$ & 0.0061 & 0.0061 & 0.0061 \\
\hline$\sigma_{V}^{\mathrm{b}}$ & 0.0074 & 0.0076 & 0.0066 \\
\hline$\sigma_{J}^{\mathrm{b}}$ & 0.027 & 0.028 & 0.027 \\
\hline
\end{tabular}

Notes.

${ }^{a}$ We report $68.3 \%$ confidence intervals, with error bars symmetrized by adopting the mean of the $15.85 \%$ and $85.15 \%$ levels as the central value.

${ }^{b}$ rms scatter of the residuals after subtracting the model from the data.

chain in order to ensure they had converged. These parameters are reported in Table 9, and Figure 3 shows the light curves with the best-fitting model overplotted.

\subsection{Discussion}

The most serious limitations in the present analysis result from the use of only single-band light curves to model the outof-eclipse variations. This is clear from comparing the results for the three solutions reported in Table 9, where we find significant ( $>1$ standard deviation) differences in the orbital inclination $i$, ratio of effective temperatures $T_{2} / T_{1}$, and in $\left(R_{1}+R_{2}\right) / a$, which depend on the assumptions made regarding the locations of the spots on the components of the binary.

Given our radial velocity errors, the orbital inclination uncertainty has little effect on the final physical parameters, as evidenced by the component masses and semimajor axis reported in Table 9, which are essentially identical to within the observational errors. The dominant uncertainty in the final solution is therefore in the component radii.

Of the three models considered, the symmetric spot distribution in model 3 seems the most physically realistic given the known properties of spots on low-mass stars. These are often found to be polar in light curve models (e.g., Rodonò et al. 1986). Moreover, Doppler imaging studies (e.g., Barnes et al. 2000; Barnes \& Collier Cameron 2001) indicate that in reality, the surfaces of low-mass stars probably have many small spots distributed over a range of latitudes. This situation is largely indistinguishable from a small number of large, polar spots in one-dimensional light curve data, although it is important to note that the effects on the measured eclipse depths could be different. There is no a priori reason to expect the spots to be concentrated into one hemisphere given the close to edge-on inclination of the binary orbit. We therefore favor model 3 , but report all three solutions to provide a realistic evaluation of the systematic error in our results, and reiterate that the data do not distinguish between the three possibilities, with all of these having comparable $\chi^{2}$ values and residuals.

As a check, we compare the model fit to the MEarth data alone with our $V$ - and $J$-band data in Figure 3 . In order to do this, we have refitted the model for these bands allowing only the light curve normalization parameter (HLA) to vary, with all other parameters fixed to the values determined from the MEarth data. The model is consistent with the $J$-band data within the scatter, and is reasonably consistent with the $V$-band data, considering that the early parts of the secondary eclipse curve were taken during quite nonphotometric conditions, and that we have not accounted for color dependent or differential atmospheric extinction effects, which are expected to be larger in this band than the redder MEarth or $J$ bandpasses, and cannot readily be determined due to the lack of repeat observations.

The presence of spots and proximity effects in very close binary systems have a small influence on the shape of the radial velocity curve, causing it to depart from a Keplerian orbit as we assumed in Section 4. We have investigated the influence of these effects on our results by comparing radial velocity curves generated using the WD model and the simple Keplerian model, finding that the corrections to radial velocities taken out of eclipse are dominated by the spots, the properties of which are largely unknown. However, these are 



Figure 3. Phase-folded light curves in MEarth, $V$, and $J$ passbands (top, middle, and bottom panels, respectively). In each panel, the upper subpanels show the light curve (black points) with the WD model 3 (see Table 9) overplotted (solid lines), and the lower subpanels show the residuals after subtracting the model from the data. The uppermost panel for the MEarth data shows a binned light curve (in 250 bins each spanning 0.004 in normalized orbital phase) to allow the features to be more clearly distinguished given the large number of data points.

(A color version of this figure is available in the online journal.)

very small $\left(<0.1 \mathrm{~km} \mathrm{~s}^{-1}\right)$ compared to the uncertainties in our radial velocity measurements (approximately $6 \mathrm{~km} \mathrm{~s}^{-1}$ ), so we are therefore justified in neglecting them for the present analysis.

\section{EFFECTIVE TEMPERATURES, LUMINOSITIES, AND KINEMATICS}

Provided the effective temperature of one component of an eclipsing binary is known, the combined light curve and radial velocity parameters are then sufficient to determine intrinsic, bolometric luminosities of both components of the system. This follows directly from the definition of the effective temperature in terms of the Stefan-Boltzmann law:

$$
L_{\mathrm{bol}}=4 \pi R^{2} \sigma T^{4} .
$$

By using bolometric corrections and the measured system magnitudes, we can then infer the distance, provided the reddening can be constrained or assumed to be zero. In the present case, the latter is a reasonable assumption, since our target is very nearby (Gliese \& Jahreiss 1991 give a "photometric parallax" of $47 \pm 8 \mathrm{pc}$, and Lépine 2005 gives $21.3 \pm 4.4 \mathrm{pc}$; note that both of them assume that the system is a single star, which means they underpredict the distance to a near equal luminosity binary such as the present one by a factor of approximately $\sqrt{2}$ ).

In order to determine the effective temperatures, we assume the intrinsic colors and bolometric corrections for M dwarfs compiled by Bessell $(1995,1991)$ and Bessell \& Brett (1988) to convert the integrated system $V-I$ color presented in Table 5 into $T_{1}$, assuming the measured effective temperature ratio and radii for both components from the light curve model. We used the polynomial fits of Hillenbrand (1997), which cover the required spectral-type range, and assume an uncertainty of 0.1 mag in these fits as stated in her Appendix C. We also assume a $\pm 100 \mathrm{~K}$ systematic uncertainty in the effective temperature scale, which has been included and propagated in the errors we 


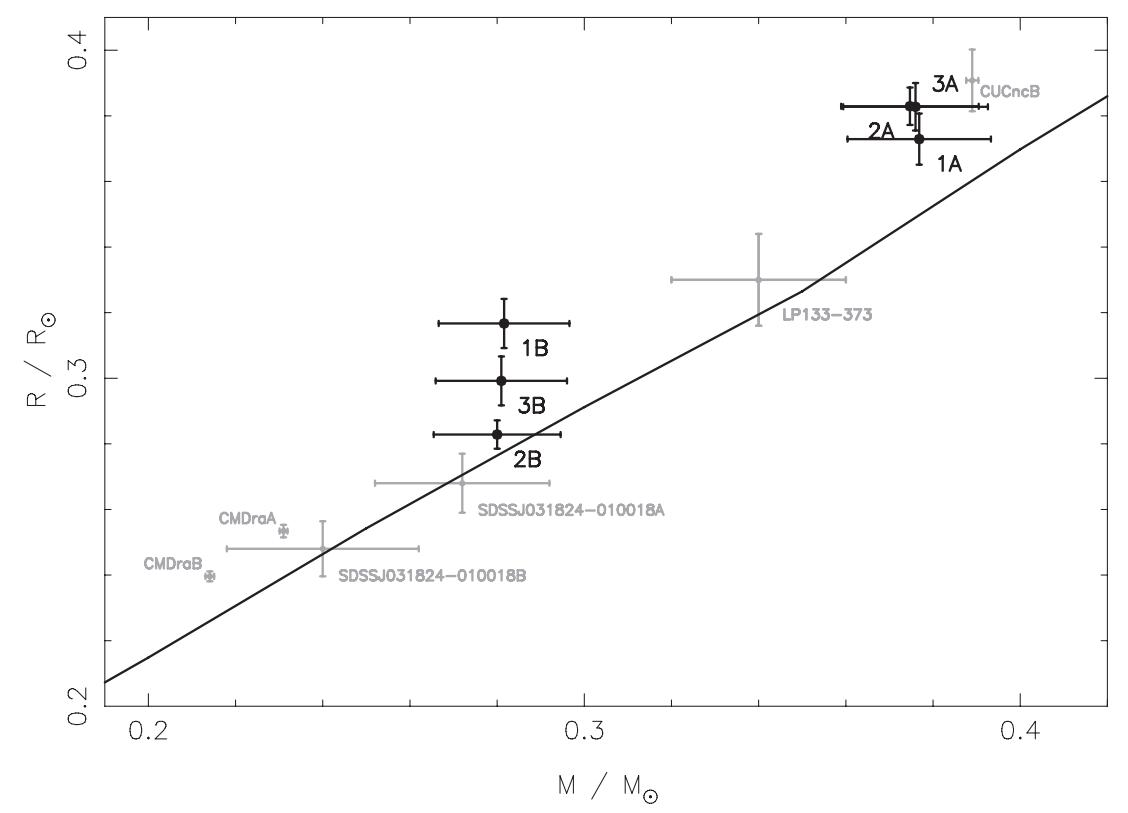

Figure 4. Mass-radius relation for eclipsing double-lined spectroscopic binary systems with one or more components below $0.4 M_{\odot}$. The black points with error bars show the two components of the GJ 3236 system, for all three solutions reported in Table 9. The gray points are known systems from the literature (Morales et al. 2009; Ribas 2003; Vaccaro et al. 2007; Blake et al. 2008). The black line shows the theoretical mass-radius relationship from Baraffe et al. (1998) for an age of 1 Gyr.

Table 10

Effective Temperatures, Luminosities, and Kinematics for GJ 3236

\begin{tabular}{lccc}
\hline \hline Parameter & \multicolumn{3}{c}{ Value } \\
\cline { 2 - 4 } & Model 1 & Model 2 & Model 3 \\
\hline$T_{1}$ & $3313 \pm 110 \mathrm{~K}$ & $3310 \pm 110 \mathrm{~K}$ & $3313 \pm 110 \mathrm{~K}$ \\
$T_{2}$ & $3247 \pm 108 \mathrm{~K}$ & $3241 \pm 108 \mathrm{~K}$ & $3238 \pm 108 \mathrm{~K}$ \\
$L_{\mathrm{bol}, 1}$ & $0.0152 \pm 0.0021 L_{\odot}$ & $0.0160 \pm 0.0022 L_{\odot}$ & $0.0160 \pm 0.0021 L_{\odot}$ \\
$L_{\mathrm{bol}, 2}$ & $0.0101 \pm 0.0014 L_{\odot}$ & $0.0080 \pm 0.0011 L_{\odot}$ & $0.0089 \pm 0.0012 L_{\odot}$ \\
$M_{V}$ & $11.17 \pm 0.30$ & $11.23 \pm 0.30$ & $11.19 \pm 0.30$ \\
$M_{I}$ & $8.44 \pm 0.27$ & $8.50 \pm 0.27$ & $8.46 \pm 0.27$ \\
$(m-M)$ & $3.11 \pm 0.28$ & $3.05 \pm 0.28$ & $3.09 \pm 0.28$ \\
$d$ & $42.2 \pm 5.5 \mathrm{pc}^{-1}$ & $41.1 \pm 5.3 \mathrm{pc}^{-1}$ & $41.8 \pm 5.4 \mathrm{pc}^{-1}$ \\
$U$ & $+34.2 \pm 3.9 \mathrm{~km} \mathrm{~s}^{-1}$ & $+33.5 \pm 3.8 \mathrm{~km} \mathrm{~s}^{-1}$ & $+34.0 \pm 3.9 \mathrm{~km} \mathrm{~s}^{-1}$ \\
$V$ & $-20.7 \pm 4.0 \mathrm{~km} \mathrm{~s}^{-1}$ & $-20.0 \pm 3.9 \mathrm{~km} \mathrm{~s}^{-1}$ & $-20.5 \pm 4.0 \mathrm{~km} \mathrm{~s}^{-1}$ \\
$W$ & $-2.5 \pm 1.7 \mathrm{~km} \mathrm{~s}^{-1}$ & $-2.4 \pm 1.7 \mathrm{~km} \mathrm{~s}^{-1}$ & $-2.5 \pm 1.7 \mathrm{~km} \mathrm{~s}^{-1}$ \\
\hline
\end{tabular}

report. Table 10 gives our derived parameters for the GJ 3236 system.

The refined value of $T_{1}$ reported here differs by approximately $35 \mathrm{~K}$ from the assumed value in Section 3 used to determine limb-darkening coefficients. This discrepancy is $<1 \sigma$ and should have a negligible effect on the parameters determined from the light curve analysis, so we elected not to repeat this using the updated value of $T_{1}$ given the computational expense involved.

Given the full system kinematic information (position, proper motions, $\gamma$ velocity, and parallax from the EB analysis), we can also calculate the $U V W$ components of the space velocity relative to the Sun. We use the method of Johnson \& Soderblom (1987), but adopt the definition that positive $U$ values are away from the Galactic center (a left-handed coordinate system) for better consistency with the literature. These are included in Table 10. Comparing to the velocity ellipsoids derived by Chiba \& Beers (2000), GJ 3236 appears to be a member of the Galactic disc and lies within the bounds of the young Galactic disc population as defined by Leggett (1992). Comparing to the $U V W$ space motions of various solar neighborhood moving groups (e.g., Soderblom \& Mayor 1993), the velocities are consistent with with those of the Hyades moving group, which has $(U, V, W)=(+38 \pm 6,-17 \pm 6,-11 \pm 12) \mathrm{km} \mathrm{s}^{-1}$ (Zhao et al. 2009). Despite this possible membership in the Hyades group, we note that the dispersion in stellar parameters for the group members does not provide a very useful constraint on age or metallicity for the present system. Zhao et al. (2009) obtain a metallicity of $[\mathrm{Fe} / \mathrm{H}]=-0.09 \pm 0.17$ for the Hyades group.

\section{DISCUSSION}

Figures 4 and 5 show the position of GJ 3236 relative to other known objects and theoretical models of the $M$ dwarf massradius and mass-effective temperature relationships.

Although many of the best-observed systems are found to exhibit larger radii than the models predict, the slope of the mass-radius relation between the components of each system (or equivalently, the ratio of the component radii) is generally found to agree well between the observations and the theoretical models. Examining the positions of our solutions for GJ 3236, our models 1 and 3 for the out-of-eclipse modulations are 


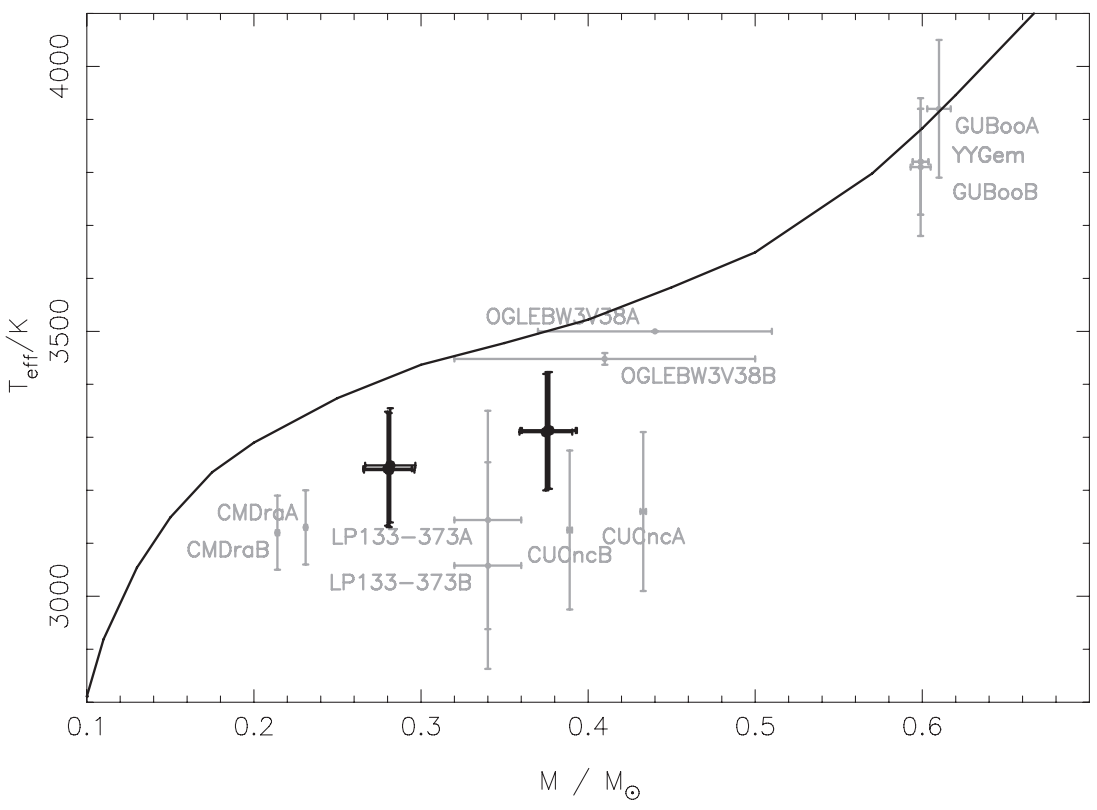

Figure 5. Same as Figure 4, but showing the mass-effective temperature relationship. Again, all three solutions are shown for GJ 3236, but the differences are relatively minor in this plane. We also include several more massive M dwarf EBs in this figure for comparison: YY Gem (Torres \& Ribas 2002), GU Boo (López-Morales \& Ribas 2005), and OGLE BW3 V38 (Maceroni \& Montalbán 2004). The black line shows the theoretical relationship from Baraffe et al. (1998) for an age of 1 Gyr.

reasonably consistent with this expectation, whereas the slope defined by the components for solution 2 is substantially different. Although this indicates the assumptions used in model 2 may be unrealistic, the present observational data for GJ 3236 do not distinguish between the three possibilities. Using this as a constraint in the modeling would be dangerous because the results would then no longer yield a completely independent test of the theoretical models, so instead we suggest that a better solution to the issue would be obtaining improved multiband photometry to better constrain the spot parameters.

Therefore, within the present observational uncertainties, the radii of GJ 3236 appear to be consistent with the theoretical predictions at approximately $1 \sigma$, when we account for the systematic uncertainties represented by the three solutions we have presented. Furthermore, the central values appear to lie above the theoretical curve. This is largely consistent with the results for the well known systems in the literature, where the radii are typically found to be $10 \%-15 \%$ larger than the theoretical predictions.

In the effective temperature versus mass plane (Figure 5), the effective temperatures of the components of GJ 3236 are found to be cooler than the models predict, by approximately $2 \sigma$. This is in good agreement with many of the well known systems in the literature as shown in the figure, such as CM Dra.

Compared to the well known systems CM Dra and CU Cnc, GJ 3236 has a shorter orbital period, and the $\mathrm{H} \alpha$ emission in the spectroscopy and out-of-eclipse modulations we observe in the light curve are indicative of high activity levels, with both the photometric period and the spectroscopic line broadening apparently consistent with the stellar spin being synchronized to the binary orbit, as expected from tidal theory (e.g., Zahn 1977).

Chabrier et al. (2007) propose two hypotheses to explain the observed radius discrepancies between theoretical models and eclipsing binaries: (1) that the inflated radii result from reduced convective efficiency due to high rotation rates and large magnetic fields; or (2) that magnetic spot coverage of the surfaces leads to reduced heat flux, and thus larger radii and cooler effective temperatures. Hypothesis (2) has been found to be consistent with some of the well known eclipsing binary systems, e.g., YY Gem (van Gent 1926; Joy \& Sanford 1926; Torres \& Ribas 2002), where the radius discrepancy (and the discrepancy in effective temperature) can be explained by the presence of starspots covering approximately $50 \%$ of the stellar surface (if the spots are cooler than the photosphere by 15\%; Ribas et al. 2008). GJ 3236 shows out-of-eclipse modulations of comparable amplitude to YY Gem, a compatible radius discrepancy within the present observational errors, and similarly has effective temperatures somewhat cooler than the models predict, so this is an attractive hypothesis. By obtaining precise, multiband photometry covering the entire orbital phase, it may be possible to constrain the spot temperatures, and hence observationally test this argument.

The main limitations in the orbital and geometric modeling of the system arise from the lack of out-of-eclipse data in multiple passbands, which leads to large errors $(4 \%-5 \%)$ on the radii when we take the degeneracies in the spot configuration into account. The precision of the mass estimates $(5 \%)$ is limited by the error in the radial velocity measurements, and an important contribution to the uncertainties in the radii will be from the spectroscopic light ratio if the uncertainty in spot parameters can be resolved. Further high-precision photometric measurements and high-resolution spectroscopy are therefore needed, and combined with careful analysis these should allow the precision of the mass and radius measurements for this system to be improved, potentially to beyond the $2 \%$ level as for the well known systems CM Dra and YY Gem.

The MEarth team gratefully acknowledges funding from the David and Lucile Packard Fellowship for Science and Engineering (awarded to D.C.). This material is based on work supported by the National Science Foundation under grant number AST-0807690. S.N.Q. and D.W.L. acknowledge support from the NASA Kepler mission under cooperative 
agreement NCC2-1390. G.T. acknowledges partial support from the NSF through grant AST-0708229. We thank Eric Mamajek for helpful discussions regarding solar neighborhood kinematics, and the "astro-comb" team at CfA: Claire Cramer, David Phillips, Alexander Glenday, Chih-Hao Li, Dimitar Sasselov and Ronald Walsworth, for their generous donation of engineering time to obtain spectra of our target. The referee is thanked for comments that helped to improve the manuscript.

This research has made extensive use of data products from the Two Micron All Sky Survey, which is a joint project of the University of Massachusetts and the Infrared Processing and Analysis Center/California Institute of Technology, funded by NASA and the NSF, NASA's Astrophysics Data System (ADS), and the SIMBAD database, operated at CDS, Strasbourg, France. The Digitized Sky Surveys were produced at the Space Telescope Science Institute under U.S. Government grant NAG W-2166. The images of these surveys are based on photographic data obtained using the Oschin Schmidt Telescope on Palomar Mountain and the UK Schmidt Telescope. The plates were processed into the present compressed digital form with the permission of these institutions. The Peters Automated Infrared Imaging Telescope (PAIRITEL) is operated by the Smithsonian Astrophysical Observatory (SAO) and was made possible by a grant from the Harvard University Milton Fund, the camera loan from the University of Virginia, and the continued support of the SAO and U.C. Berkeley. The PAIRITEL project and J.S.B. are further supported by NASA/Swift Guest Investigator Grant NNG06GH50G. We thank M. Skrutskie for his continued support of the PAIRITEL project.

\section{REFERENCES}

Andersen, J. 1991, A\&AR, 3, 91

Baraffe, I., Chabrier, G., Allard, F., \& Hauschildt, P. H. 1998, A\&A, 337, 403 Barnard, E. E. 1916, AJ, 29, 181

Barnes, J. R., \& Collier Cameron, A. 2001, MNRAS, 326, 950

Barnes, J. R., Collier Cameron, A., James, D. J., \& Donati, J.-F. 2000, MNRAS, 314,162

Bessell, M. S. 1991, AJ, 101, 662

Bessell, M. S. 1995, in The Bottom of the Main Sequence Any Beyond, ed. C. Tinney (Berlin: Springer), 123

Bessell, M. S., \& Brett, J. M. 1988, PASP, 100, 1134

Blake, C. H., et al. 2005, Nature, 435, 181

Blake, C. H., Torres, G., Bloom, J. S., \& Gaudi, B. S. 2008, ApJ, 684, 635

Bloom, J. S., Starr, D. L., Blake, C. H., Skrutskie, M. H., \& Falco, E. E. 2006, in ASP Conf. Ser. 351, Astronomical Data Analysis Software and Systems XV, ed. C. Gabriel et al. (San Francisco, CA: ASP), 751

Çakirli, Ö., İbanoğlu, C., \& Güngör, C. 2009, New Astron., 14, 496

Chabrier, G., Gallardo, J., \& Baraffe, I. 2007, A\&A, 472, 17

Chiba, M., \& Beers, T. C. 2000, AJ, 119, 2843

Claret, A. 2000, A\&A, 363, 1081

Claret, A. 2004, A\&A, 428, 1001

Delfosse, X., Forveille, T., Mayor, M., Burnet, M., \& Perrier, C. 1999, A\&A, 341,63

Eggen, O. J., \& Sandage, A. 1967, ApJ, 148, 911
Etzel, P. B. 1981, in Photometric and Spectroscopic Binary Systems, ed. E. B. Corling \& Z. Kopal (Dordrecht: Reidel), 111

Ford, E. B. 2005, AJ, 129, 1706

Gliese, W., \& Jahreiss, H. 1991, Preliminary Version of the Third Catalogue of Nearby Stars (Heidelberg: Astron. Rechen Institut)

Hastings, W. K. 1970, Biometrika, 57, 97

Hauschildt, P. H., Allard, F., \& Baron, E. 1999, ApJ, 512, 377

Hebb, L., Wyse, R. F. G., Gilmore, G., \& Holtzman, J. 2006, AJ, 131, 555

Hewett, P. C., Irwin, M. J., Bunclark, P., Bridgeland, M. T., Kibblewhite, E. J., He, X. T., \& Smith, M. G. 1985, MNRAS, 213, 971

Hillenbrand, L. A. 1997, AJ, 113, 1733

Irwin, J., Charbonneau, D., Nutzman, P., \& Falco, E. 2009, in IAU Symp. 253, Transing Planets, ed. F. Pont, D. Sasselov, \& M. Holman (Cambridge: Cambridge Univ. Press), 37

Irwin, J., Irwin, M., Aigrain, S., Hodgkin, S., Hebb, L., \& Moraux, E. 2007a, MNRAS, 375, 1449

Irwin, J., et al. 2007b, MNRAS, 380, 541

Johnson, D. R. H., \& Soderblom, D. R. 1987, AJ, 93, 864

Joy, A. H., \& Sanford, R. F. 1926, ApJ, 64, 250

Kopal, Z. 1959, Close Binary Systems (New York: John Wiley \& Sons)

Kurucz, R. L. 1979, ApJS, 40, 1

Kurucz, R. L. 1993, VizieR On-line Data Catalog, VI/39

Kwee, K. K., \& van Woerden, H. 1956, Bull. Astron. Inst. Neth., 12, 327

Lacy, C. H. 1977, ApJ, 218, 444

Landolt, A. J. 1992, AJ, 104, L340

Leggett, S. K. 1992, ApJS, 82, 351

Lépine, S. 2005, AJ, 130, 1680

Lépine, S., \& Shara, P. 2005, AJ, 129, 1483

López-Morales, M. 2007, ApJ, 660, 732

López-Morales, M., \& Ribas, I. 2005, ApJ, 631, 1120

Lucy, L. B. 1967, Z. Astrophys., 65, 89

Maceroni, C., \& Montalbán, J. 2004, A\&A, 426, 577

Metcalfe, T. S., Mathieu, R. D., Latham, D. W., \& Torres, G. 1996, ApJ, 456, 356

Metropolis, N., Rosenbluth, A. W., Rosenbluth, M. N., Teller, A. H., \& Teller, E. 1967, J. Chem. Phys., 21, 1087

Morales, J. C., et al. 2009, ApJ, 691, 1400

Nutzman, P., \& Charbonneau, D. 2008, PASP, 120, 317

Popper, D. M., \& Etzel, P. B. 1981, AJ, 86, 102

Prša, A., \& Zwitter, T. 2005, ApJ, 628, 426

Ribas, I. 2003, A\&A, 398, 239

Ribas, I., Morales, J. C., Jordi, C., Baraffe, I., Chabrier, G., \& Gallardo, J. 2008, Mem. Soc. Astron. Italiana, 79, 562

Rodonò, M. 1986, A\&A, 1986, 165, 135

Soderblom, D. R., \& Mayor, M. 1993, AJ, 105, 226

Southworth, J., Maxted, P. F. L., \& Smalley, B. 2004a, MNRAS, 351, 1277

Southworth, J., Smalley, B., Maxted, P. F. L., Claret, A., \& Etzel, P. B. 2005, MNRAS, 363, 529

Southworth, J., Zucker, S., Maxted, P. F. L., \& Smalley, B. 2004b, MNRAS, 355,986

Tody, D. 1993, in ASP Conf. Ser. 52, Astronomical Data Analysis Software and Systems II, ed. R. J. Hanisch, R. J. V. Brissenden, \& J. Barnes (San Francisco, CA: ASP), 173

Torres, G., \& Ribas, I. 2002, ApJ, 567, 1140

Vaccaro, T. R., Rudkin, M., Kawka, A., Vennes, S., Oswalt, T. D., Silver, I., Wood, M., \& Smith, J. A. 2007, ApJ, 661, 1112

van Gent, H. 1926, Bull. Astron. Inst. Netherlands, 3, 121

Wilson, R. E., \& Devinney, E. J. 1971, ApJ, 166, 605

Zahn, J.-P. 1977, A\&A, 57, 383

Zhao, J., Zhao, G., \& Chen, Y. 2009, ApJ, 692, 113

Zucker, S., \& Mazeh, T. 1994, ApJ, 420, 806 